\title{
Reconstruction of lateral bone defects according to Khoury's biologic concept : a clinical and radiological evaluation of 30 consecutive cases.
}

\author{
Dr. Trasarti S. ${ }^{1}$, Dr.ssa Marroni D. ${ }^{1}$, Dr.Braun I. ${ }^{2}$ \\ 1) Privat Office Teramo and Maccagno (Va) - Italy \\ 2) Privat Office Froendberg - Germany
}

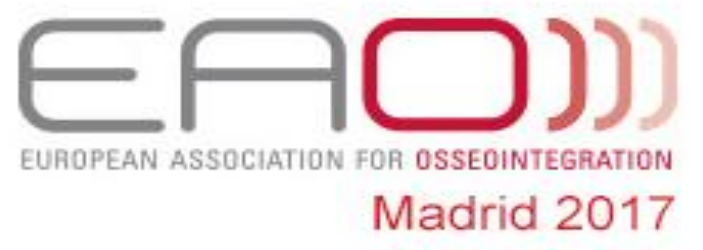

Background: In the last years, the fixed restoration with dental implants has become increasingly widespread, due to the predictability and the development of implant therapy. This has generated in the patient an increase of expectations as for the aesthetic and the function. Often the surgeon has to treat situations that require an increase of the bone in order to place the implants in a correct prosthetic position. Today only the autogenous bone gives guarantee and predictability in the long term.

Aim:. The present study evaluated the results of 30 cases of lateral bone augmentations in the anterior maxilla reconstructed according to the Khoury's biologic concept with the "splitted bone block technique". The purpose was to register the gained bone thickness and it's stability up to 5 years.

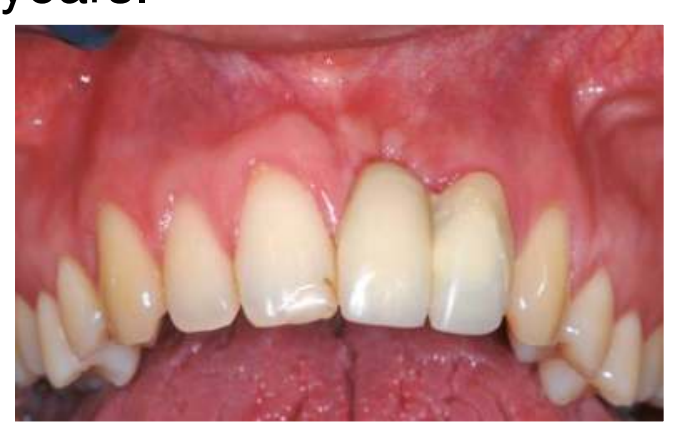

Preoperative situation

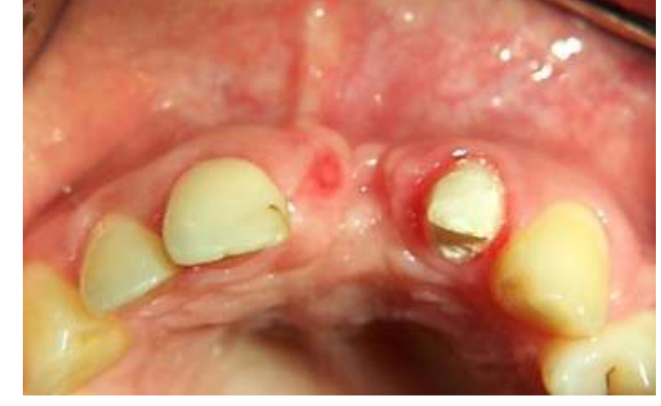

Occlusal view
Results: A total of 30 patients ( 21 female, 9 male) were treated with this procedure in the anterior area of the maxilla. The primary bone width was $1-3 \mathrm{~mm}$ and increased at the end of the surgery to an average of $7,5 \mathrm{~mm}$. The thickness of the grafted crest 3 months post operative had an average of $6,6 \mathrm{~mm}$ so that all the planned 53 implants were successfully inserted. No healing problems occurs. All the implants healed as expected and were restored after an average of 4 months. A total of 21 Patients received additionally a connective tissue graft during implant exposure. The patients were regularly controlled between 3 and 5 years without any implant failure. Two patient drop out after 3 years. All the patients presented a satisfied functional and aesthetics result. The radiographic control documents the stability of the grafted and regenerated bone over the years.
Materials and methods: Before to proceed to bone harvesting, it was required to all the patients an orthopantomogram and a CBCT to register anatomical landmarks and to estimate the bone atrophy. The bone block was harvested from the mandibular retromolar area with the Microsaw technique under antibiotic prophylaxis and then splitted longitudinally into two blocks. Bone chips were gained by scraping the blocks until a thickness of $1 \mathrm{~mm}$. The blocks were screwed on distance to remaining crest. The space between the residual bone and the blocks was filled with the scraped bone chips. Three months after the procedure the implants were inserted and after other 3 months exposed with an apical repositioned flap and restored.

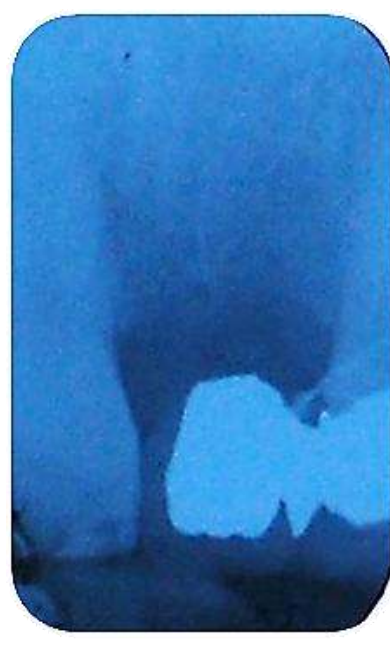

Rx pre OP

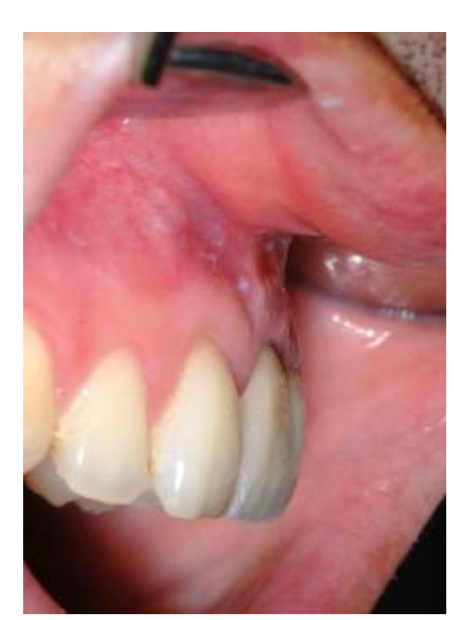

After 7 years

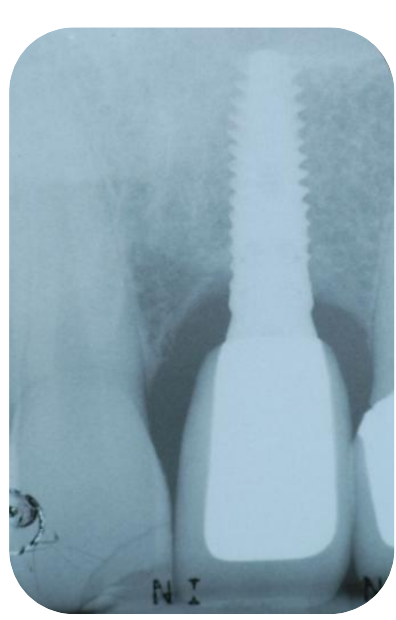

$\mathrm{RX}$ after 7 years

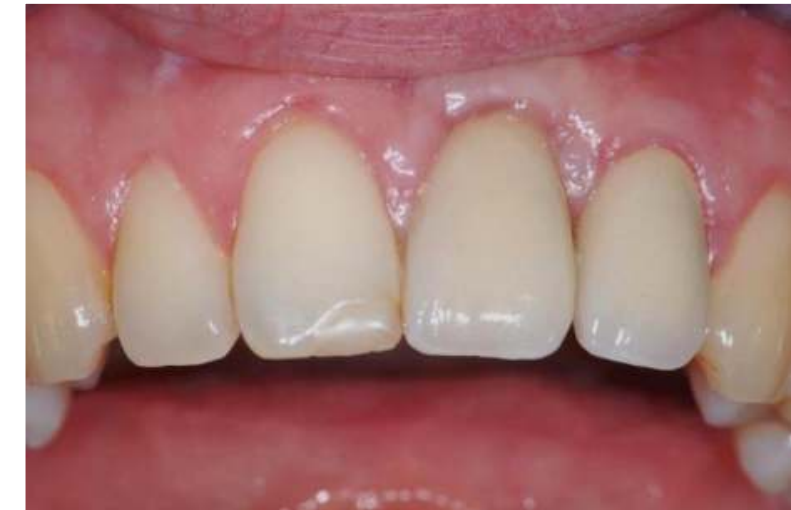

View after 7 years of loading

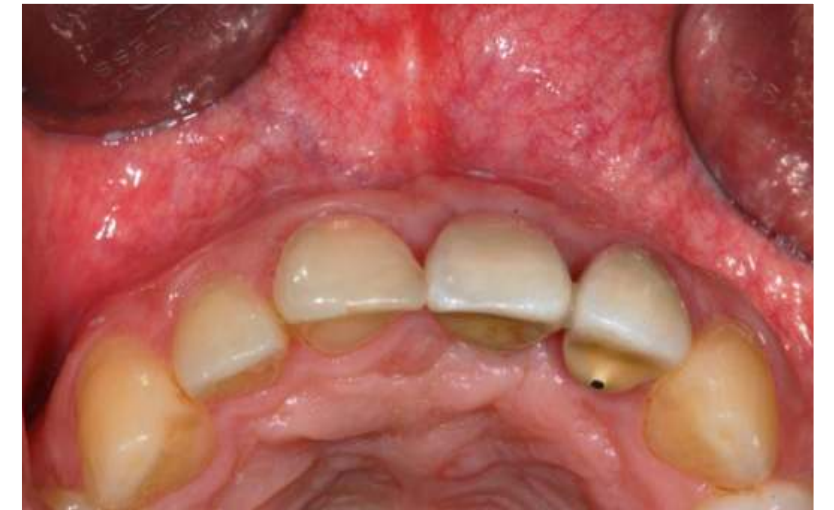

Occlusal view after 7 years
Conclusion: Patients with severe lateral bone loss treated with autogenous bone blocks according to the biological concept showed stable results up to 5 years post operative.

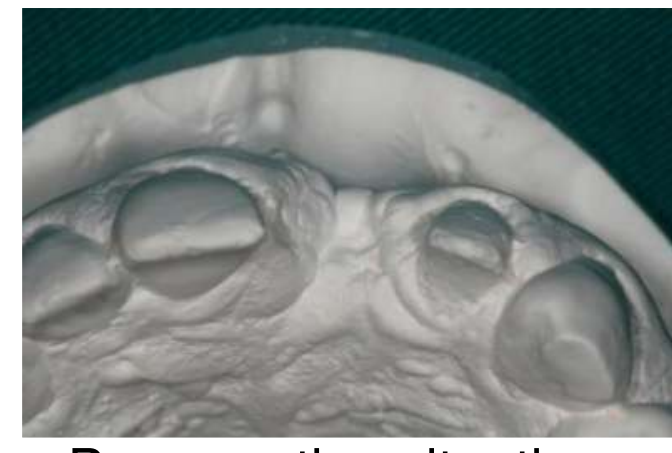

Preoperative situation

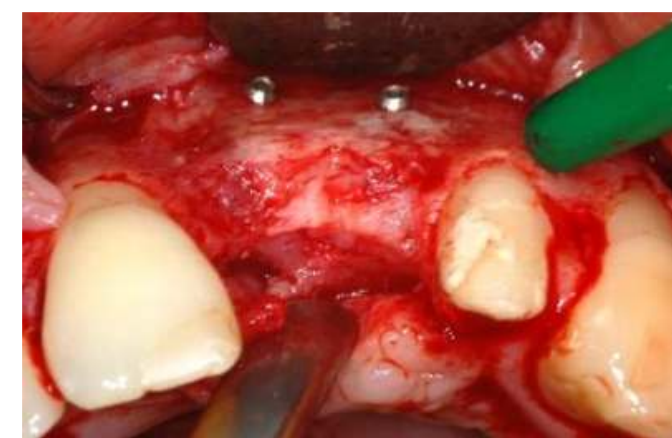

Situation after 3 months

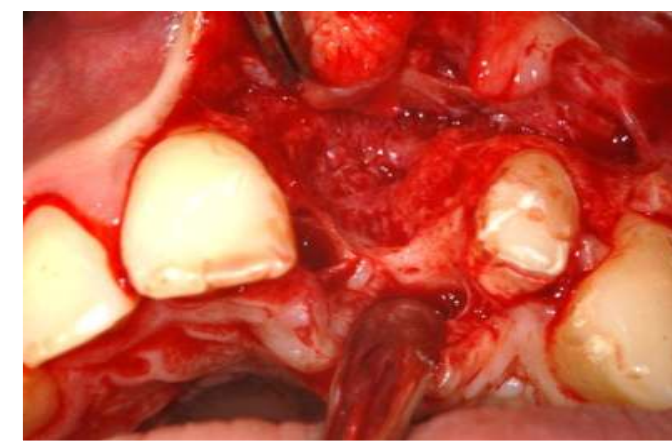

Horizontal defect

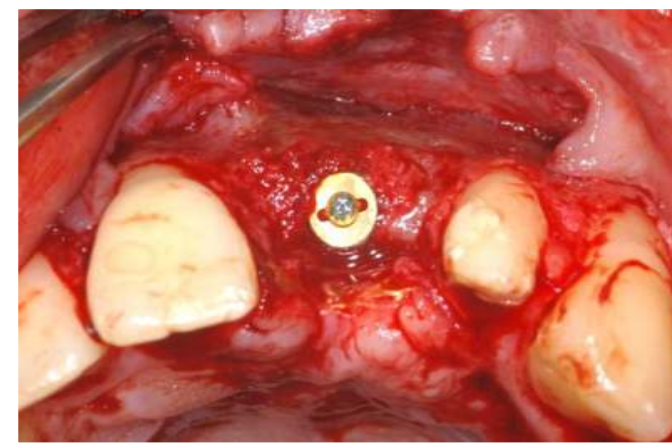

Implantation

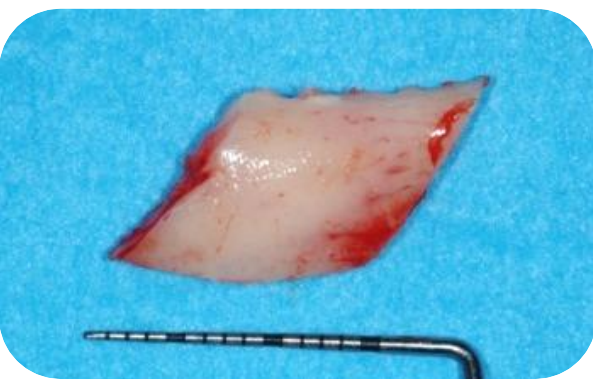

Harvesting bone block

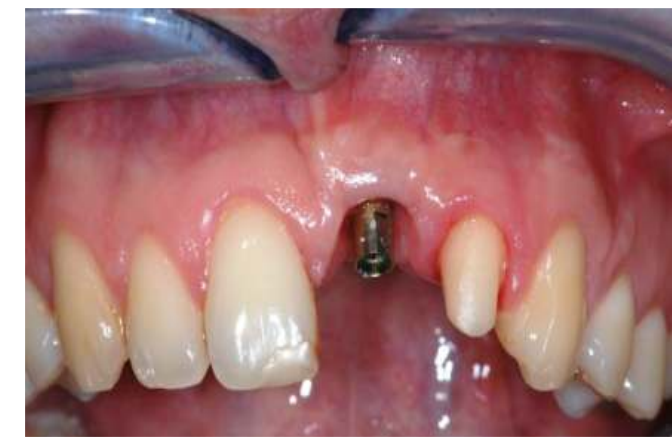

Healing after soft tissue management

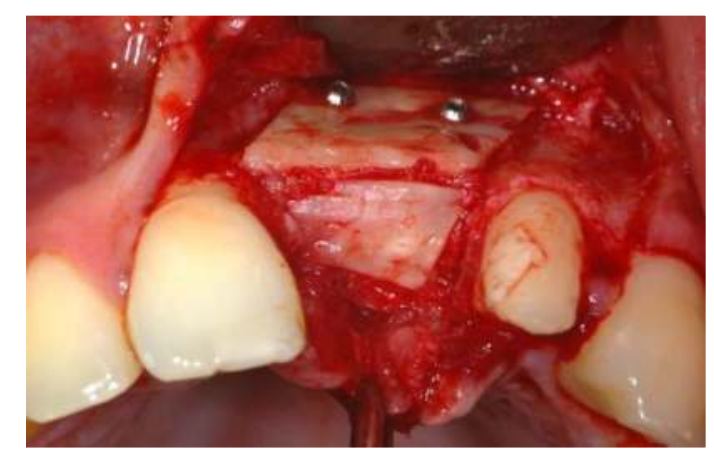

$3 \mathrm{D}$ bone reconstruction
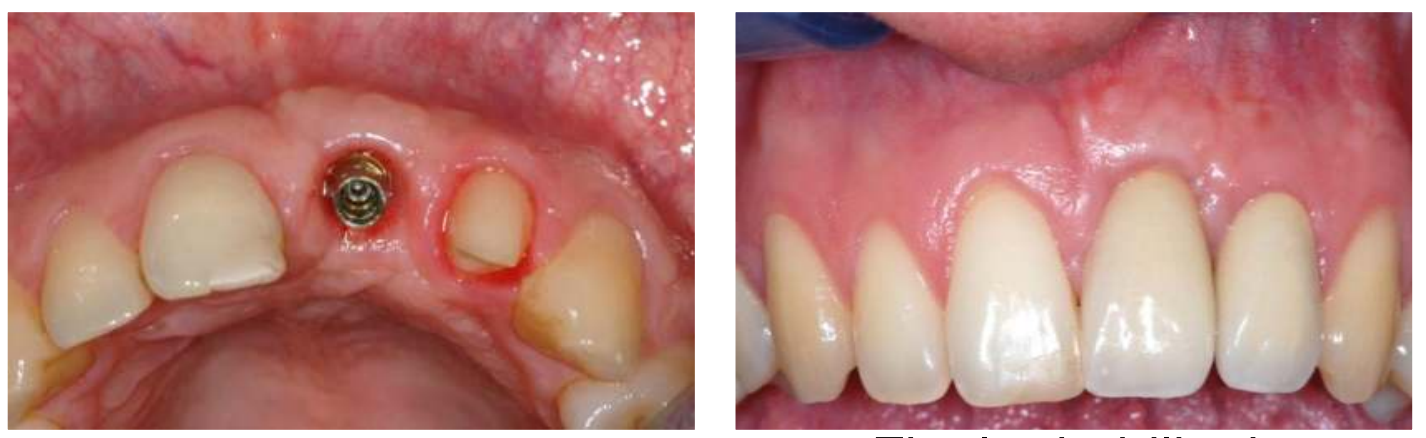

Final rehabilitation

\section{Bibliography}

1 Khoury F, Khoury C. Mandibular bone block grafts: instrumentation, harvesting technique and application. Journal de Parodondologie \& d'Implantologie Orale 2006;25:15-34.

2 Khoury $F$ et al: Bone augmentation in oral implantology. Quintessence Publishing 2007

3 Trasarti S, Khoury $F$ «Autogenous bone blocks harvesting from the mandibular retromolar area: a clinical report of 160 clinical consecutive treated patients. European Association of Osteointegration Paris 2016

4 Trasarti S., Marroni D., Braun I.: Simultaneous implant placement and 3D bone reconstruction with autogenous bone-follow-up after four years. Z Zahnärztl Impl / 2016; 32 (2) 